\title{
Teaching Engineering Conferences
}

\author{
Thomas Zielke, Matthias Neef, and Claudia Fussenecker \\ Düsseldorf University of Applied Sciences (HSD) \\ thomas.zielke@hs-duesseldorf.de
}

\begin{abstract}
This article is on a university course called Engineering Conferences. It has been developed by the authors and installed as a mandatory part of the curriculum in three master's degree programs for engineering students. The participants of the course are postgraduates with different nationalities, mostly German, and different technical backgrounds. They study Mechanical Engineering, Simulation and Experimental Technology, or International Business Engineering.

The basic idea of the course concept goes far beyond simply teaching the standards of academic writing and skills for using scientific publications. By using a learnercentered approach, the students get engaged in typical activities around an active attendance of a real conference. Students learn to locate the field of their bachelor thesis or project report in the world of research communities, scientific journals and engineering conferences. They learn about conferences matching their bachelor/project topic. They write a paper complying with common academic standards, submit it to a mock-up conference, and review submissions of their fellow students. Students also produce a poster and have to defend it in a poster session held publicly on the campus. Engineering Conferences is a course on scientific communication and presentation that also aims at the development of other skills and competences needed in the world of modern engineering.
\end{abstract}

Keywords: engineering curricula, soft skills, student research, scientific communication, interdisciplinary teaching, active learning

\section{INTRODUCTION}

\subsection{Engineering Curricula}

Engineering curricula are traditionally dominated by technical teaching contents. This typically comprises factual knowledge, mathematical and engineering methods, phenomenological theories, fundamental design concepts, use of quantitative data and procedures for achieving some specified aim whether that involves designing a product or service or solving a problem [29]. Very often, curricula have evolved over many years, regularly being fitted to the expertise of the faculty's teaching staff. Curricula of this kind have long been criticized for the lack of essential skills and attributes that are required for engineers in the 21st Century. As Nguyen argues in a 1998 article [27], engineering curricula should be designed to develop all the essential skills and attributes, which could be established by considering the following points:

- the level of acquired knowledge of an engineer

- the necessary skills of an engineer

- the job requirements of an engineer

- personal and professional attributes of an engineer

- attitude of an engineer

Today, it is even more evident that many curricula need reforms in that direction $[19,3]$. However, changes are happening slowly, and engineers take pride in their classic strengths to the extent that some engineering curricula themselves are designed by an engineering methodology [31, 32].

The starting point for the work described in this article was the design of five new bachelor's degree programs and three new master's degree programs at the faculty of mechanical and process engineering of a medium-sized university of applied sciences in Germany.

\section{2 "Soft Skills" for Engineering Students}

According to common standards of engineering education, our course Engineering Conferences is about "soft skills". This term is a familiar one in faculty meetings on engineering curricula, a remarkable fact as there is little consensus on what this term actually means and hardly anybody uses the term "hard skills". In faculty discussions, a lack of students' soft skills is often complained about by teachers that encounter operational difficulties with their students in running classes on some hard skills. Apart from that, discussions are rare about skills that are outside the engineering domains defined by the study programs. Although there are numerous publications on soft skills in engineering education, e.g. $[39,30,33,6]$, a precise definition of soft skills can not be found. Engineers are tempted to think of soft skills as non-technical skills (cf. [27]). But what's about "digital literacy" [11], for example? This is a common term for describing a set of competencies and technical skills that increasingly overlap with the elements of a reasonable 
general education. Another example for the complexity of the debate about soft skills and hard skills is given by Piczak and Heidebrecht [29]. They convincingly propose to teach "wisdom" as a particular soft skill. This could be incorporated into curricula by means of an "embedded model" or a "competency based model". Wisdom is an obvious example for a soft skill that very often outperforms hard skills at solving engineering problems.

For the purpose of this discourse, Levasseur [22] offers helpful orientation on the nature of soft skills. He recognizes the following categories:

- personal skills, e.g. self-awareness

- interpersonal skills, e.g. effective communication

- group skills, e.g. the ability to work in teams

- organizational skills, e.g. leadership competences

\subsection{The "Aspect Space" of Modern Engineering Education}

What skills and competencies really matter in today's engineering jobs? A survey by the Eastern Kentucky University, USA, asked business executives to list the 10 most important soft skills they wanted new employees to possess when hired for a position within their organization. As a major result of this study, Robles summarizes that "soft skills are just as good an indicator of job performance as traditional job qualifications (hard skills)" [30]. Indeed, the academic distinction between hard and soft skills does not seem to matter from the employers' point of view. The international recruitment service Monster answers the question "What skills are Engineering employers looking for?" on its UK website [1]. "Regardless of the role in which you will be working, there are a common set of intangible skills that employers look for across all engineering disciplines": Effective communication skills, interpersonal skills, technical knowledge, organizational skills, enthusiasm, and commitment. This is almost a complete repetition of what we cited above about soft skills. The point is that modern engineering education requires both teachers and students to accept that engineering professionals must possess a set of skills and competencies which cannot be subdivided and classified into "hard", i.e. important but difficult to learn, and "soft", i.e. inessential and seemingly easy to learn. Non-engineering work plays a larger role in jobs than engineering students expect and the faculties do not realize that a large proportion of all engineering graduates eventually work in non-engineering-related jobs [4].

"A modern day engineer is likely to be part of a multidisciplinary, multi-cultural, globally dispersed team" [21]. No doubt, working in engineering has become multidimensional, to use a technical term. In a modern vision, engineering education is multi-dimensional too, covering a primarily technical dimension and additional dimensions described as key aspects by Aldert Kamp of the Delft University of Technology [19]. This is not in contradiction to the parallel trend of technical specialization. The acquisition and development of core knowledge and capabilities in the domain of the engineering sciences become strongly focused while more space is created for other aspects.

1. Rigor of engineering

2. Critical thinking and unstructured problem solving

3. Interdisciplinary and systems thinking

4. Imagination, creativity, initiative

5. Communication and collaboration

6. Global mind-set: diversity and mobility

7. Ambitious learning culture: student engagement and professional learning community

8. Employability and lifelong learning

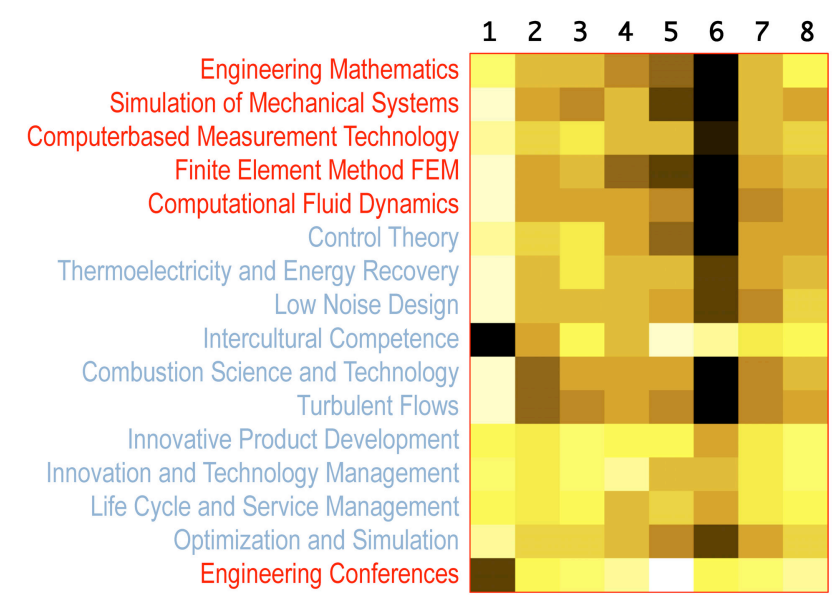

Fig. 1. The key aspects of engineering education as stated by Kamp [19], numbered from 1 to 8 (upper part). Lower part: The courses offered to the master students in Mechanical Engineering (red: mandatory / blue: elective specializations) juxtaposed with a matrix of weights that show a rough estimate of the contributions a course makes with respect to the key aspects. White stands for the highest score, black for the lowest. The authors independently made estimates for the scores. The illustration shows the average values of the three estimates.

In an "aspect space" of engineering education each course of a study program can contribute to one or more aspects in different ways and to different degrees. We analyzed the curriculum of our master's program "Mechanical Engineering" as to what extent the individual courses may contribute to different aspects of modern engineering education. Figure 1 shows a juxtaposition of the courses offered to the master students in Mechanical Engineering and the key aspects as formulated by Kamp. It has to be said, for the design of this curriculum, the different aspects relevant for modern engineering education were not explicitly taken into account. Therefore the analysis shown in Fig. 1 should be seen as a basis for further improvements of the 
curriculum. As stated by Aslaksen [3] "the present approach of adding a few non-technical subjects to the engineering curriculum is not effective". However, with Engineering Conferences we want to tune our master's degree programs significantly towards "the future of engineering" [3].

\section{THE ROLE OF RESEARCH IN ENGINEERING STUDY PROGRAMS}

Undergraduate students in engineering often have little exposure to the world of scientific publishing and the culture of sharing research work. Only postgraduate students working towards a $\mathrm{PhD}$ are confronted with the necessity to publish and to learn from the publications of other researchers. While some research oriented universities offer undergraduate research programs and special journals for undergraduate publications [18, 35], bachelor theses at teaching oriented universities often display significant deficiencies concerning the standards of technical/scientific writing.

One goal of Engineering Conferences is to create awareness for academic standards in research and development and to equip students with basic publication skills. As only a relatively small proportion of all graduates think of taking a job in research, it is not unusual that students ask "Do I need research skills in working life?" [25]. One may also ask why the aspect space of engineering education, described in section 1.2, does not contain explicit references to research work. Both questions are misleading. By doing research work and by active participation in a research community, skills and competencies related to most aspects of a good engineering education are acquired and developed, as illustrated in Figure 2.

Research and teaching have at least co-existed if not cross-fertilized each other for centuries, although it has been suggested and surveyed that there is little statistical evidence for a correlation between the two [13]. Today, there is an increasingly severe conflict of objectives. University teachers face great pressure to publish papers in internationally indexed journals, often leading to a neglect of other academic activities, including teaching [34]. On the other hand, it can be very beneficial for students when their teachers have substantial research experience and ongoing research activities, see section 5 . The problems have to do with the value and the judgment of research quality in general which is currently challenged by governmental influence to develop highlevel research in selected universities, see [18]. For the objective of good engineering teaching, it may be helpful to remember the medieval meaning of the word research: "go about seeking" [17]. There is no shortcut for students from being involved in this activity to become self-reliant learners. Ideally, research activities are embedded in undergraduate curricula and courses in a way to enhance student learning $[36,38,15]$. Students can also be encouraged to voluntarily attend graduate student conferences, including the submission of their project work for publication [7]. At the latest, students gain some sort of research experience during their bachelor thesis, which therefore can be regarded as research in their field of study - regardless of quality and outcome. In a nutshell, research is learning, and learning is research and should be supported by teaching [26].

We therefore regard the bachelor thesis of each student as an existing piece of research and take it as a starting point for the course on engineering conferences within the master's degree programs of our faculty. Our concept follows a research-based approach [14], focusing on the research process rather than on the research content, which has already been dealt with during the thesis. In this process, the students become participants rather than an audience.

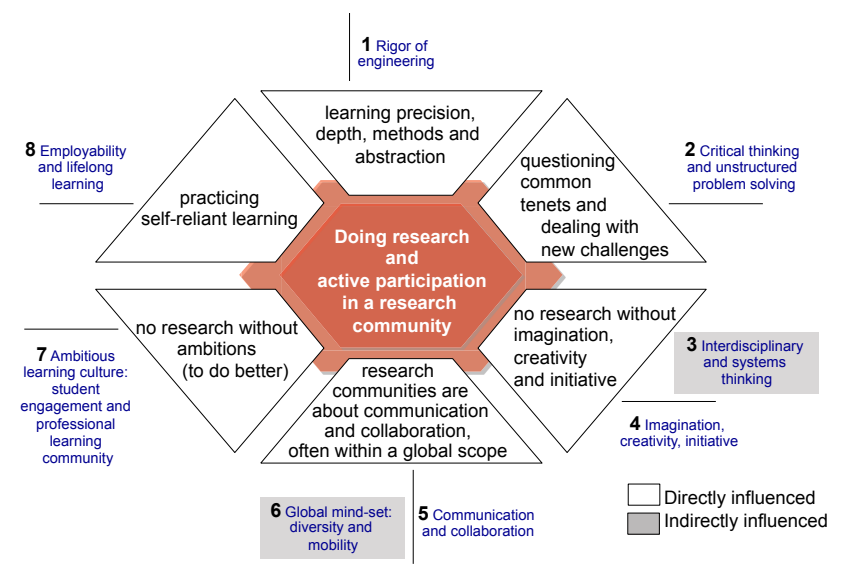

Fig. 2. Doing research and active participation in a research community is training for skills and competencies related to most aspects of a good engineering education.

\section{THE COURSE FRAMEWORK AND THE EXAMINATION PLAN}

Beyond teaching the standards of academic writing and skills for using scientific publications, we want to get the students engaged in typical activities around an active attendance of a real conference. By doing this, most of the important aspects of modern engineering education should be addressed either explicitly or implicitly.

For the design of our course, a few publications provide valuable orientation, concrete ideas and "lessons learned", e.g. [23] and [24]. One experience from running our course for the very first time in the winter term 2016/2017 has been the importance of firstly the examination plan, i.e. what do the students have to deliver for getting good grades, and secondly the means for raising the motivation of the students for this course. 
From student feedback we had to realize that many of the participants thought of Engineering Conferences as a course on dispensable soft skills that only matter to those considering a PhD study after the master's degree. Studies by Benson et al. [5] show that the motivation of the students for a particular course within their study program depends significantly on the students' perceptions of their future tasks as engineers. In the current course schedule we therefore start the term with a session entirely devoted to Motivation.

Table 1 provides the data of the course Engineering Conferences as part of the master's curricula in our faculty. Figure 3 shows its structure. Basically there are two phases: Training and Practice. The objectives of the training phase are a general understanding of the world of academic research and scientific communication, as well as gaining experience in professional reflection and selfreflection. There are no examination elements in this phase. The course elements of the training phase can be called Motivation, Orientation, Comprehension, and Classification \& Interrelation.

Table 1: Course Details - Facts and Figures.

\begin{tabular}{|l|l|}
\hline Module Name: & Engineering Conferences \\
\hline $\begin{array}{l}\text { Master Program } \\
\text { (3 semesters): }\end{array}$ & $\begin{array}{l}\text { Mechanical Engineering, } \\
\text { Simulation and Experimental } \\
\text { Technology, International Business } \\
\text { Engineering }\end{array}$ \\
\hline Module Type: & Mandatory \\
\hline Credits / Workload: & $\begin{array}{l}\text { 6 ECTS (European Credit Transfer and } \\
\text { Accumulation System) / 180 hours }\end{array}$ \\
\hline Language: & EMI (English as a means of instruction) \\
\hline Exam Elements: & $\begin{array}{l}\text { paper, two paper reviews, poster } \\
\text { presentation }\end{array}$ \\
\hline Semester of Attendance: & first, second or third \\
\hline Number of Participants: & 30 per term \\
\hline
\end{tabular}

The practice phase focuses on course elements that are part of or belong to a simulated engineering conference. Whenever applicable, "EasyChair" (easychair.org) is used to organize our simulated conference and the activities associated with it. The elements Publishing Tools \& Standards and Paper Concept deal with preparations for the publication of an article on a piece of research, concretely the bachelor thesis. The other elements of the practice phase are part of a simulation of a real conference. The "grand finale" of the course is the poster session held publicly on the campus. The Poster Presentation therefore is the examination element with the highest weight, currently $60 \%$ of the total grade. The other examination elements are the paper submission $(20 \%)$ and the delivery of two reviews of papers submitted by fellow students $(10 \%$ each).

The course element Conference Session serves two purposes. First, the students are given the experience of an oral session of a conference. Second, the Conference Session allows students who have already been authors of a real conference paper, accepted by peer-review, to present their paper to the class. Those students can opt to earn their credits from the recognition of their publication experience. At the same time, the other students can draw motivation from realizing that a peer student actually succeeded with a submission of a paper at a real engineering conference. In any case, the Conference Session also contains talks by invited speakers, usually researches from our faculty.

Poster Preparation and Poster Presentation are given the highest weight in the examination plan. For most students, these two course elements also carry the highest workload compared with the other elements. Our focus on the poster as the "main product" of the course has at least three good reasons: (1) At quality conferences, most junior researchers actually present in a poster session. (2) The learning benefits from working on conference posters and poster presentations have been well documented [37, $20,8]$. (3) A poster can serve as a kind of masterpiece, something physical the students can take home and be proud of.

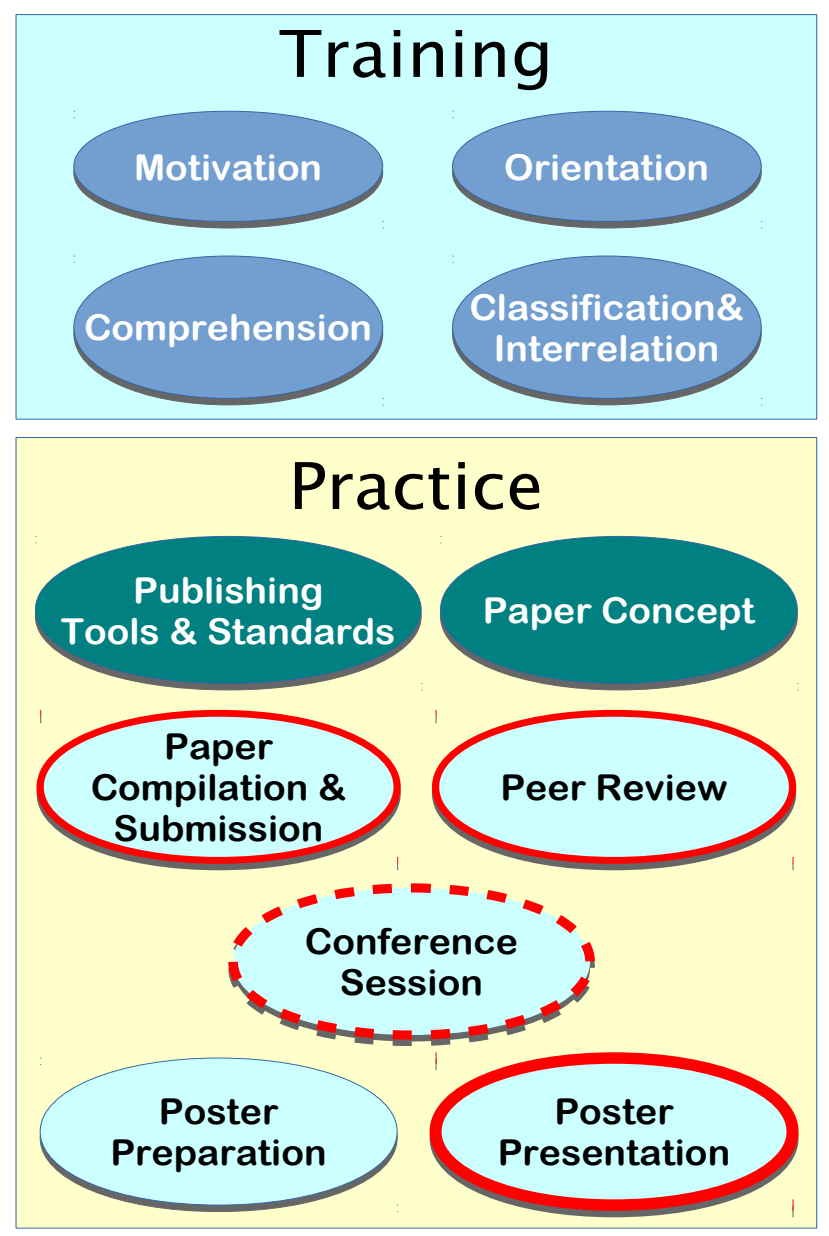

Fig. 3. The general course structure and the elements of Engineering Conferences. Elements that are relevant for grading are marked with a red outline. 


\section{THE COURSE ELEMENTS - ACTIVE LEARNING}

The previous section describes the framework for the course Engineering Conferences. The individual course elements can be designed and put into practice in different ways. We actually intent to optimize and/or vary the course elements from term to term, as more student feedback and practical experience become available.

The general teaching approach is based on the concept of Active Learning [10]: "Active learning engages students in the process of learning through activities and/or discussion in class, as opposed to passively listening to an expert. It emphasizes higher-order thinking and often involves group work."

A detailed description of each course element of Engineering Conferences is beyond the scope of this paper. However, we want to briefly describe some ideas, techniques, approaches, and topics that we found to be useful for the students and well received by them.

\subsection{Motivation}

Increasing the motivation of the students for the course is a subject of the kickoff session. We ask the students to write down keywords for what they expect to learn by the course. The keywords are clustered on a pinboard. Typically, groups of expectations like "improvement of English language skills", "learning to summarize a lot in less words", or "improvement of presentation skills" emerge. This provides a link to a lecture on the importance of this kind of skills and competencies for the future jobs of the participants. Actually, the introduction for this article contains the message that this lecture wants to convey: Doing research and active participation in a research community is a great training for many skills and competencies that are needed in engineering jobs.

\subsection{Orientation}

For their bachelor thesis, students usually work on a very specific problem. They often do not realize the likeliness that other researchers worked on the same problem, somewhere in the world. For novices in a field it is difficult to even recognize the parallels between similar projects if the language and/or the technical terms of the projects' titles are different. Novices in the world of sciences are lost and they need orientation.

We have developed an explorative activity by which students can find fellow students that worked in a similar technical/scientific field for their bachelor thesis. The idea was inspired by a project of Hassan-Montero et. al [12]. On the website www.scimagojr.com/shapeofscience/ almost 19,000 scientific journals are represented by dots on a map which is called The Shape of Science. The size of a dot indicates the importance (rank) of a journal and there is much more information about the journals linked with the dots. The vicinity of two journals on that map expresses the degree to which the articles in these journals are interrelated.
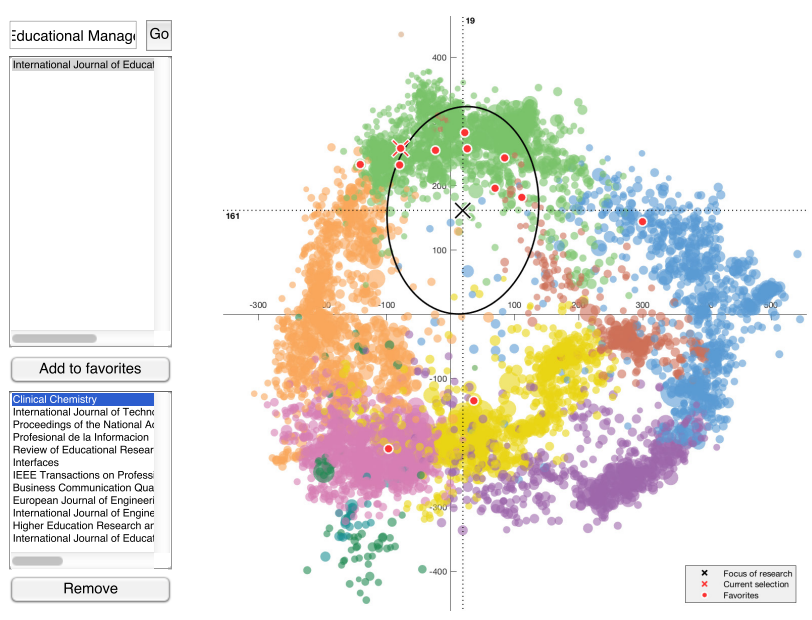

Fig. 4. The list of journals cited by a publication creates an elliptic region on the Shape of Science map. The center of this region is the "research focus" of this publication on a map of subject areas. The example shown was generated from the journals referenced in this paper.

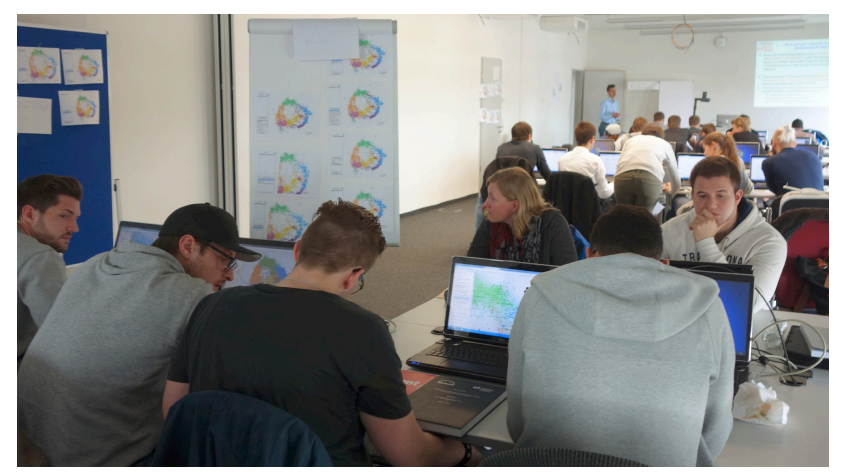

Fig. 5. The class of summer term 2017 working on the Shape of Science activity.

The students are given the task to collect all journal references from their bachelor thesis. They are asked to browse The Shape of Science for the journal names. Then they are given a visualization tool 1 developed for Engineering Conferences on the basis of the data available from The Shape of Science. As shown in Figure 4 , this tool creates an elliptic region on the Shape of Science map for the list of journals collected from a publication. We call the center of this region a "research focus". Finally, every student prints out the map showing his/her research focus. All maps are put on pinboards and the students split up into distinct "research communities" by comparing their maps and discussing similarities and distinctions of their research. The newly established 
groups, the research communities within the class, are a basis for subsequent group work.

\subsection{Comprehension / Classification\&Interrelation}

Many graduates in engineering are not accustomed to the use of scientific publications for their work. Most of our students also feel uneasy when being confronted with scientific/technical texts written in English. The same applies to professional oral communication using the English language. We have to meet our students at this point. Comprehension needs communication. This should be relatively easy within a group of "experts" in the same field, e.g. within our research communities formed within the class. The group work starts with Elevator Talks [2], each being a 1-minute opportunity for a group member to explain the topic of her/his bachelor thesis to the others. The next task has to be worked on by the whole group. It is a structured review and analysis of an article that the group can freely select from a reputed journal in their field.

Classification \& Interrelation is about the techniques and the knowledge needed for a comprehensive literature research. The ability to efficiently classify publications as potentially relevant and important is an essential skill in the present-day (digital) world of scientific and technical publications. For novices in a scientific field, discovering interrelations between one's own work and that of others is an additional challenge. As an objective of the practical course work under this topic, all students redo and improve the literature research for their bachelor thesis.

\subsection{Publishing Tools \& Standards}

There are many books and articles on scientific paper writing, $[16,9]$, just to mention two exceptionally useful examples. Within the scope of Engineering Conferences we have to concentrate on teaching the basics and supporting "learning by doing" by constructive critical feedback. In any case, students must know essential standards and what benefits the use of professional tools can provide. Additionally, the teachers have to convey awareness for quality and professionality, in the sense of an ambitious learning culture. As an example, we briefly describe the teaching content on citation rules and reference management. Students must know that:

- Acknowledging the sources of information and ideas is essential, not something optional.

- There are several widely used referencing styles, but in every publication one style has to be used consistently.

- Each conference or journal asks for the use of one particular style, usually a document template is provided.
- Correct citations are required for all types of sources: internet, lecture notes, TV programs, videos etc., all can be cited properly [28].

- Usually, references do not have to be typed in manually, a complete citation in several formats is often provided by the publisher, generation via the Digital Object Identifier (DOI) is a convenient option too.

- For all projects that eventually result in a report, thesis, or some sort of publication, a reference management system should be used.

The practical exercises for the teaching/learning session on citation rules and reference management use the open source software JabRef (jabref.org). As with other tools, we prefer software that all students can work with on their private computers free of charge and without commercial obligations. At the same time, we try to make students understand the general concepts and features of a software species, things that can also be used with other products. After completing the exercises the students have the list of references of their bachelor thesis in a BibTex database.

\section{DISCUSSION AND CONCLUSIONS}

We have developed and implemented a course on scientific communication and presentation for the master's degree programs at the faculty of mechanical and process engineering of a medium-sized university of applied sciences in Germany. In their bachelor study, most of our students have not been confronted with the necessity to publish and/or to learn from research publications. Only few students are fluent in written and spoken English. As the name Engineering Conferences suggests, the course is designed around a mock-up conference, where students have to present the results of their bachelor thesis as a poster. The combination of the following features distinguishes the course from similar approaches:

- There is a storyline (conference preparation) and a public final (presentation day).

- The students are assumed to have had the experience of a substantial technical project, usually the bachelor thesis, but no experience with scientific publications.

- The students are engaged as researchers, turning the publication of their thesis into a project.

- The course is mandatory for all master students of the faculty.

- The teachers are from within the faculty and have extensive experience as active researchers.

- The course concept can be easily copied and integrated into any master's degree program in engineering. 
Engineering Conferences is the first course of its kind at our university. The design and the first implementation of the course has been a challenge and a learning experience for the authors, as the teaching objectives do not match any of their usual teaching domains. However, it turned out that practical experience in engineering, teaching experience in classical engineering subjects, an active involvement in research, being author of articles in scientific journals, and having published at engineering conferences are the prerequisites for running a course like the one described in this article.

While our course concept may serve as a blueprint for other engineering faculties, we also put this course in the context of modern engineering education. We argue that student participation in research is vital for reaching the objectives of engineering education. This participation should be accompanied by courses like Engineering Conferences but the research activities themselves should become an integral part of many courses in the engineering curricula [36].

Within the framework described in section 3 there is room and flexibility for future course improvements, in particular as to the effectiveness and the range of active learning methods. We also need to find reliable methods of evaluating the contributions of the course to the success of our master's degree programs, in terms of the qualification of our master's graduates, their employability, and their satisfaction.

\section{Acknowledgements}

The authors want to thank Jens Lippel for the development of the visualization tool for the Shape of Science activity.

\section{References}

[1] "What skills are Engineering employers looking for?", 2017, April 30, Retrieved from

https://www.monster.co.uk/career-advice/article/whatskills-are-engineering-employers-looking-for

[2] Thomas M. Annesley, "The abstract and the elevator talk: a tale of two summaries", Clinical chemistry, 56(4): 521-524, March 2010

[3] Erik W. Aslaksen, "The future of engineering", in Journal and Proceedings of the Royal Society of New South Wales, volume 148, pages $159-165$. Royal Society of New South Wales, 2015.

[4] Beryl L. Benderly, "Checkered careers", Prism Magazine, American Society for Engineering Education (ASEE), January 2015.
[5] Lisa Benson, Adam Kirn, and Courtney Faber, "Career: Student motivation and learning in engineering", in ASEE Annual Conference Proceedings, 2013.

[6] Aseel Berglund and Fredrik Heintz, "Integrating soft skills into engineering education for increased student throughput and more professional engineers", in LTHs 8: e Pedagogiska Inspirationskonferens, Lund, Sweden, 17 December 2014. Lund University, 2014.

[7] William C. Brown, "Developing professionally through graduate student publications", In Progress, 2:77-88, 2012.

[8] Ayelet Cohen and Jenny McDonald, "From paste- up to power-up: Supporting students to design a research poster", in Proceedings of the 27th Annual Conference of the Australasian Society for Computers in Learning in Tertiary Education, ASCILITE 2010, pages 206-210, 2010.

[9] Michael Derntl, "Basics of research paper writing and publishing", International Journal of Technology Enhanced Learning, 6(2): 105-123, 2014.

[10] Scott Freeman, Sarah L. Eddy, Miles McDonough, Michelle K. Smith, Nnadozie Okoroafor, Hannah Jordt, and Mary P. Wenderoth, "Active learning increases student performance in science, engineering, and mathematics", in Proceedings of the National Academy of Sciences, 111(23): 8410-8415, 2014.

[11] Jean-Marie Gilliot, Serge Garlatti, and Gwendal Simon, "Impact of digital literacy on the engineering curriculum", in Proceedings of the 6th International CDIO Conference, Ecole Polytechnique, Montreal., June 2010.

[12] Yusef Hassan-Montero, Vicente P. Guerrero-Bote, and F. de Moya-Anegon, "Graphical interface of the scimago journal and country rank: An interactive approach to accessing bibliometric information", El profesional de la informacion, 23(3): 272 - 278, 2014.

[13] John Hattie and Herbert W. Marsh, "The relationship between research and teaching: A meta-analysis", Review of educational research, 66(4): 507 - 542, 1996.

[14] Mick Healey and Alan Jenkins, "Developing undergraduate research and inquiry", The Higher Education Academy, York, UK, June 2009.

[15] Mick Healey, Alan Jenkins, and John Lea, "Developing research-based curricula in college-based higher education", The Higher Education Academy, York, UK, 2014

[16] Bodil Holst and Jorge Cham, "Scientic Paper Writing - A Survival Guide". CreateSpace Independent Publishing Platform, 2015. 
[17] Merriam Webster Inc. Origin and etymology of research. www.merriam-webster.com/dictionary/research.

[18] Alan Jenkins and Mick Healey, "Undergraduate research and international initiatives to link teaching and research", Council on Undergraduate Research Quarterly, 30(3): 36 - 42, 2010.

[19] Aldert Kamp, "Engineering education in the rapidly changing world: Rethinking the mission and vision on engineering education at TU Delft", Technical report, TU Delft, Faculty of Aerospace Engineering, October 2014.

[20] Andreas Karatsolis, "Assessing visual literacy: The case of poster presentations", in Proc. IEEE Int. Professional Communication Conf., pages 1 - 7, October 2012.

[21] Klara Kövesi and Robin Clark, "Trends and issues for engineering education in the UK", in $5^{\text {th }}$ Int. Studies of Management and Organisations Conference, 2015.

[22] Robert E. Levasseur, "People skills: Developing soft skills - a change management perspective", Interfaces, 43(6): 566 - 571, December 2013.

[23] J. A. Leydens and B. M. Olds, "Publishing in scientific and engineering contexts: A course for graduate students tutorial", IEEE Transactions on Professional Communication, 50(1): 45 - 56, March 2007.

[24] Rodica I. Lung. Preparing students for presenting their research. A literature survey. Journal of Teaching English for Specific and Academic Purposes, 4(2): 287 - 292, 2016.

[25] Mari Murtonen, Erkki Olkinuora, Päivi Tynjälä, and Erno Lehtinen, "'Do I need research skills in working life ?": University students' motivation and difficulties in quantitative methods courses", Higher Education, 56(5): $599-612,2008$.

[26] Matthias Neef, Thomas Zielke, and Claudia Fussenecker, "Engineering conferences - an innovative course for master students in engineering", in Proceedings of the ETALEE 2017 Conference, Odense, Denmark. The Danish Network for Engineering Education (IUPN), May 2017.

[27] Duyen Q. Nguyen, "The essential skills and attributes of an engineer: A comparative study of academics, industry personnel and engineering students", Global Journal of Engineering Education, 2(1): 65 - 75, 1998.

[28] The University of Queensland, "Referencing style guides", 2017, April 30, Retrieved from https://web.library.uq.edu.au/research-toolstechniques/referencing/referencing-style-guides

[29] Michael Piczak and Arthur Heidebrecht, "Teaching wisdom and other soft skills within engineering curricula", in Proceedings of the Canadian Engineering Education Association, 2015.
[30] M. M. Robles, "Executive perceptions of the top $10 \mathrm{soft}$ skills needed in today's workplace", Business Communication Quarterly, 75(4): 453 - 465, October 2012.

[31] Otto Rompelman and Erik De Graaff, "The engineering of engineering education: curriculum development from a designer's point of view", European Journal of Engineering Education, 31(2): 215 - 226, May 2006.

[32] D.W. Ruth and M.G. Britton, "Curriculum as an engineering design problem", in Proceedings of the Canadian Engineering Education Association, 2011.

[33] Thomas J. Siller and John Durkin, "University-industry partnership to develop engineering students' professional skills", International Journal of Engineering Education, 29(5): 1166 - 1171, 2013.

[34] Mei Tian, Yan Su, and Xin Ru, "Perish or publish in china: Pressures on young chinese scholars to publish in internationally indexed journals", Publications, 4(2): 9, 2016.

[35] Helen Walkington, Jennifer Hill, and Pauline E. Kneale, "Reciprocal elucidation: a student-led pedagogy in multidisciplinary undergraduate research conferences", Higher Education Research \& Development, pages 1 14, 2016.

[36] Helen Walkington and Alan Jenkins, "Embedding undergraduate research publication in the student learning experience", Brookes eJournal of Learning and Teaching, 2(3): 2, 2008.

[37] Steve E. Watkins, Les L. Kinsler, Julia L. Morse, and Douglas R. Carroll, "Organizing a student poster session in an ASEE section conference", in ASEE Annual Conference and Exposition, Conference Proceedings, American Society for Engineering Education (ASEE), 2014.

[38] Cynthia M. Webster and Jacqueline Kenney, "Embedding research activities to enhance student learning", International Journal of Educational Management, 25(4): 361 - 377, May 2011.

[39] Rosetta Ziegler, "Student perceptions of "soft" skills in mechanical engineering", in International Conference on Engineering Education - ICEE 2007, Coimbra, Portugal, volume 7, September 2007. 